\title{
Preparation of Electrically Conductive Adhesives Modified with $\mathrm{AgNO}_{3}$
}

\author{
Pavel Mach \\ Department of Electrotechnology, Faculty of Electrical Engineering, CTU Prague, \\ Prague, Czech Republic \\ mach@,fel.cvut.cz
}

\begin{abstract}
The goal of the work has been to find if addition of $\mathrm{Ag}^{+}$ions into electrically conductive adhesive can improve its electrical conductivity. $\mathrm{Ag}^{+}$ions can aggregate and form additional conductive bridges between filler particles and decrease electrical resistivity of adhesive this way. $\mathrm{Ag}^{+}$ions have been formed by dissociation of $\mathrm{AgNO}_{3}$. Adhesives formed of bisphenol epoxy resin and silver flakes (one component and two components types) have been used for experiments. Two ways have been used for modification of adhesives with $\mathrm{AgNO}_{3}$ : nitrate has been added in adhesive and dissociated during curing and annealing of adhesive, or nitrate has been dissociated in ethanol at first and this solution has been mixed up into adhesive. It has been found an improvement of electrical conductivity of two components adhesive after this modification.
\end{abstract}

\section{INTRODUCTION}

Electrical parameters of electrically conductive adhesives are worst in comparison with electrical parameters of solders.

The reason of this difference is that mechanisms of electrical conductivity in alloys and adhesives are different. Whereas electron-phonon mechanism is dominant in solders, tunneling dominates in electrically conductive adhesives. Electrical conductivity in adhesives strongly depends on quality of contacts between filler particles.

Of this reason the resistivity of adhesives is substantially higher, by two or three orders usually, than resistivity of solders. Nonlinearity of the current vs. voltage characteristic and noise are higher, too. High resistivity of adhesive joints can influence function of electric circuit (e.g. when a bobbin is connected using adhesive joints, the joints resistances increase its serial resistance and decrease its quality factor). Nonlinearity and noise can be significant limiting factors for the use in higher frequencies. Nonlinearity can cause cross modulation and products of this modulation can cause additional distortion of a signal.
Therefore great effort is paid to decrease resistivity of electrically conductive adhesives.

\section{METHODS TESTED FOR IMPROVEMENT OF ELECTRICAL CONDUCTIVITY OF ECAS}

Following ways for improvement of electrical conductivity of electrically conductive adhesives have already been tested and are described in literature:

\& Higher concentration of filler particles in adhesive improves electrical conductivity of adhesive, but growths worse mechanical properties. There is a limit, when mechanical properties of adhesive joints are unsatisfactory. Such the limit is, for adhesives with isotropic electrical conductivity, based on the type of insulating matrix. As for bisphenol epoxy resin filled with $\mathrm{Ag}$ flakes the limit is approximately $80 \%$ (wt.) of Ag.

Addition of conductive particles of other types, e.g. conductive nanoparticles, or carbon nanotubes, into adhesive. Aside from the price, electrical properties of such adhesives are usually worst in comparison with adhesives without modification [1], on the other hand, if carbon nanotubes are used as additional filler, the mechanical 
properties of adhesive joints are significantly improved.

Addition of nanoparticles can be carried out by three ways:

$\tau$ The nanoparticles (nanoballs or carbon nanotubes) are added into the standard adhesive with isotropic electrical conductivity, the total content of $\mathrm{Ag}$ (or carbon) in adhesive is increased. The amount of added nanoparticles can not be too high (some percents only) due to the decrease of mechanical properties of the adhesive joints if Ag nanoballs are added and due to the increase of adhesive viscosity if carbon nanotubes are added.

$\ddots$ The nanoparticles substitute some part of silver microflakes in electrically conductive adhesive with isotropic electrical conductivity. Mechanical properties are improved usually (especially when carbon nanotubes are used as additional filler), however, electrical properties are worst in comparison with adhesive without modification. The reason is significant increase of number of contacts between filler particles.

. Fabrication of nanoadhesive. Electrical properties of nanoadhesives are worst than electrical properties of standard electrically conductive adhesives filled with microflakes. The reason is the same like in the previous case. A significant increase of contacts between filler particles.

The goal of the work has been to find if addition of Ag+ ions into standard adhesive (bisphenol epoxy resin filled with Ag flakes, concentration of flakes has been $75 \%$ wt.) will improve its electrical conductivity. The idea of this modification has been as follows:

$\succ \mathrm{AgNO}_{3}$ will be mixed into adhesive. $\mathrm{Ag}^{+}$ions will be formed by thermal dissociation of $\mathrm{AgNO}_{3}$ during adhesive curing and additional annealing.

$\succ$ Second tested way of $\mathrm{Ag}+$ ions addition into adhesive has been dissociation of $\mathrm{AgNO}_{3}$ in ethanol and addition this solution into adhesive.

The principle of positive contribution of $\mathrm{Ag}+$ ions to the electrical conductivity of adhesive is shown in Fig. 1. Ag+ ions aggregate and create additional bridges, which create more compact conductive net in adhesive and improve its electrical conductivity. These bridges do not substitute the contacts between the flakes, but they create parallel by-passes.

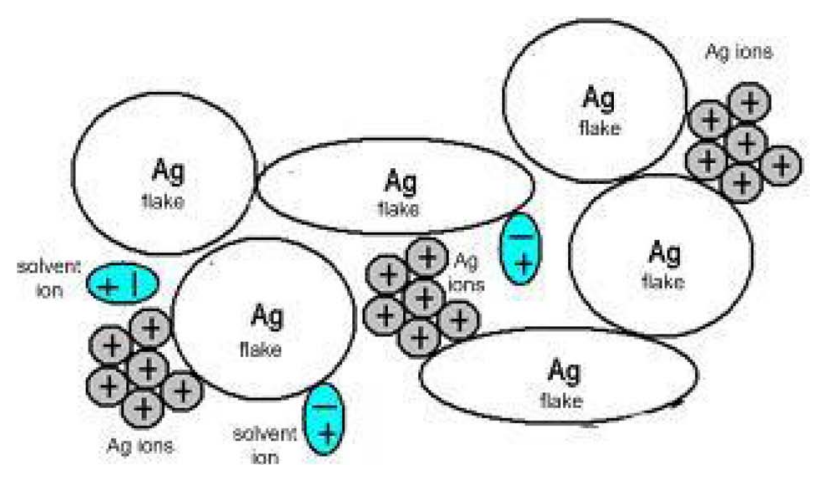

Fig. 1 Additional bridges between silver flakes created by joined $\mathrm{Ag}^{+}$aggregated ions

Attractive Van der Waals forces (these forces act on a very low distance only - approx. $10 \mathrm{~nm}$ ), repulsive Coulomb forces (see Fig. 2) and inconsiderable forces of the resistance of a matrix exist in electrically conductive adhesives. The Coulomb forces and the resistance of a matrix act against the formation of a conductive net, because they create an energetic barrier between the conductive particles.

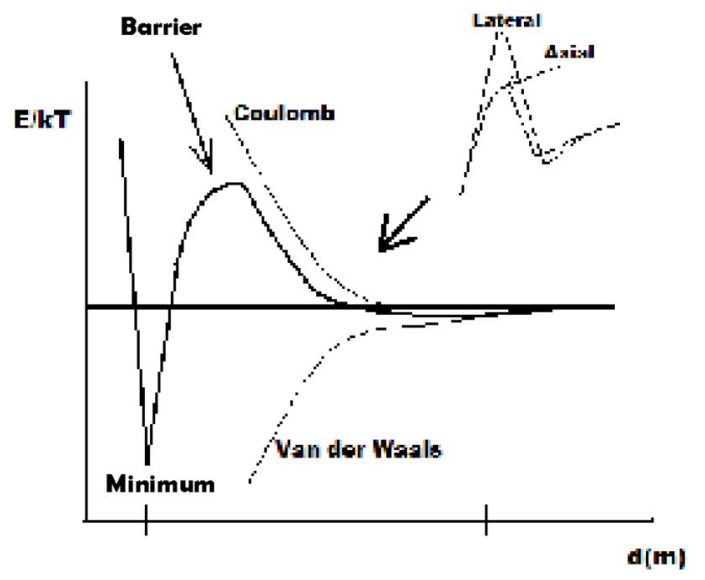

Fig. 2 Dependence of interaction energy on the particles distance 
The barriers can be surpassed using and external shear force, which can be created by rotary mixing of adhesive, or by the use of higher $\mathrm{Ag}^{+}$ions concentration in adhesive. When concentration of $\mathrm{Ag}^{+}$ ions is increased over some limit, then as a result of heating of adhesive during its curing, the ions start to aggregate.

There are two processes running during adhesive curing:

4. Ion polymerization of a polymer matrix.

¿. Aggregation of filler particles.

Ion polymerization is caused by an initiator. Elecrophilic initiator polarizes double bond of a monomer and origin carbonyl cations. This way cation polymerization is initiated.

The ion concentration of $\mathrm{Ag}^{+}$ions in polymer increases by dissociation of $\mathrm{AgNO}_{3}$ and the new cations help to overcome electrostatic forces between conductive particles (flakes), which can easier aggregate. If the temperature of adhesive increases, ions origin and support aggregation of silver flakes.

Thinner used in adhesive is vaporized during adhesive curing and its molecules are broken down from the surface of conductive particles. This way the surface charge of conductive particles decreases and probability of their aggregation increases. Simultaneously $\mathrm{Ag}^{+}$ions can join with $\mathrm{Ag}$ conductive particles, aggregate with them and create conductive bypasses between them. The structure of adhesive with added $\mathrm{AgNO}_{3}$ is shown in Fig. 3 .

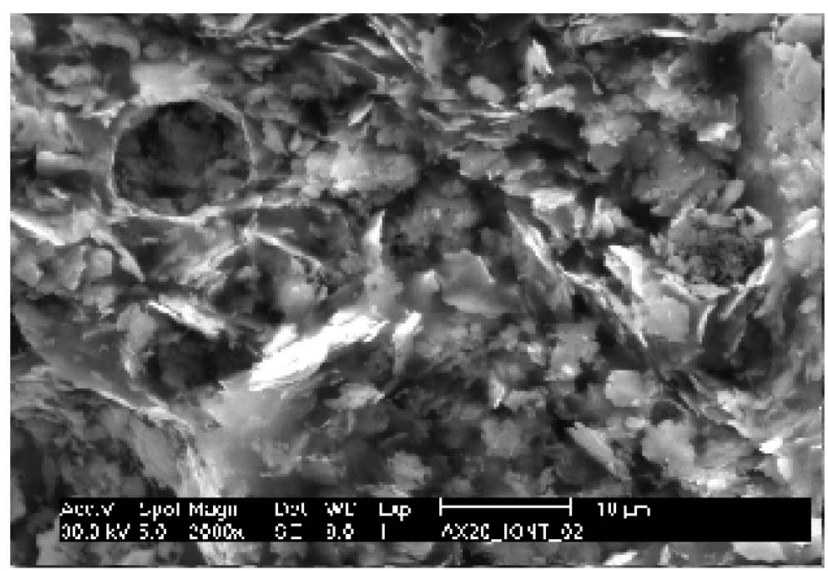

Fig. 3 Structure of adhesive with added $\mathrm{AgNO}_{3}$

\section{EXPERIMENTAL}

Electrically conductive adhesives formed of bisphenol epoxy resin, filed with silver flakes, have been used for experiments (one component and two components types). Adhesives have been modified with $\mathrm{AgNO}_{3}$. First series of experiments has been carried out with $\mathrm{AgNO}_{3}$ mixed up directly into adhesive, second series with $\mathrm{AgNO}_{3}$ first dissociated in ethanol and then added, as a solution, into adhesive.

The concentration of $\mathrm{AgNO}_{3}$ in adhesive has been found as follows:

$$
\begin{aligned}
& \mathrm{C}\left(\mathrm{AgNO}_{3}\right)=1,2 * 10^{-5} \mathrm{~mol} / \mathrm{g} \\
& \mathrm{M}\left(\mathrm{AgNO}_{3}\right)=108+14+3 * 16=170 \mathrm{~g} * \mathrm{~mol}^{-1}
\end{aligned}
$$

$$
m\left(\mathrm{AgNO}_{3}\right)=\mathrm{C}^{*} \mathrm{M}
$$

Here:

C ... requested concentration of $\mathrm{AgNO}_{3}$ in formulation, this value has been chosen $1,2 * 10^{-5} \mathrm{~mol} / \mathrm{g}$

$\mathrm{M}$... molar mass of $\mathrm{AgNO}_{3}$,

m ... calculated mass of $\mathrm{AgNO}_{3}$ in $1 \mathrm{~g}$ of adhesive

$\mathrm{AgNO}_{3}$ has been weight and added into the adhesive. Then the mixture has been mixed in an ultrasound mixing machine for $45 \mathrm{~s}$. The formulations have been used for fabrication of test specimens during next 2 hours.

Specimens have been formed of two types of adhesives - of one component and two components adhesive - as pastilles with cast-in electrodes making the four-point electrical resistivity measurement possible. The diameter of the pastilles has been $12 \mathrm{~mm}$ and the height $3 \mathrm{~mm}$. The specimen is schematically shown in Fig. 4. Electrodes have been gold wires with the diameter of $0.5 \mathrm{~mm}$.

Resistivity $\rho$ of adhesive can be calculated using a formula:

$$
\rho=\frac{U}{I} b K_{s} K_{r} K_{t}
$$

Here:

$U$... voltage measured between the opposite voltage electrodes.

I ... current flowing between the current electrodes. 
$b \ldots$ thickness of a specimen.

$K_{s} \ldots$ correction factor dependent on the diameter of the measured specimen and on the distance of the voltage electrodes. It has been calculated that $K_{s}=$ 2,9289 .

$K_{r} \quad \ldots$ correction factor dependent on the thickness of the specimen and on the distance of the voltage electrodes. It has been calculated that $K_{r}=0,9214$.

$K_{t} \ldots$ correction for the temperature. This factor is calculated using the formula:

$$
K_{t}=1+0,01(t+24)
$$

Here:

$$
\mathrm{t} \text {... temperature }\left({ }^{0} \mathrm{C}\right)
$$

The conductivity $\sigma$ has been calculated using the formula:

$$
\sigma=\rho^{-1}
$$

The four-point measurement of the resistivity has been carried out using two current electrodes and two pairs of the voltage electrodes. The voltage has been measured between opposite electrodes. Two pairs of the voltage electrodes have been used to verify homogeneity of the measurement. The measuring current $25 \mathrm{~mA}$ has been used for the measurement. The specimen used for the measurement is shown in Fig. 4.

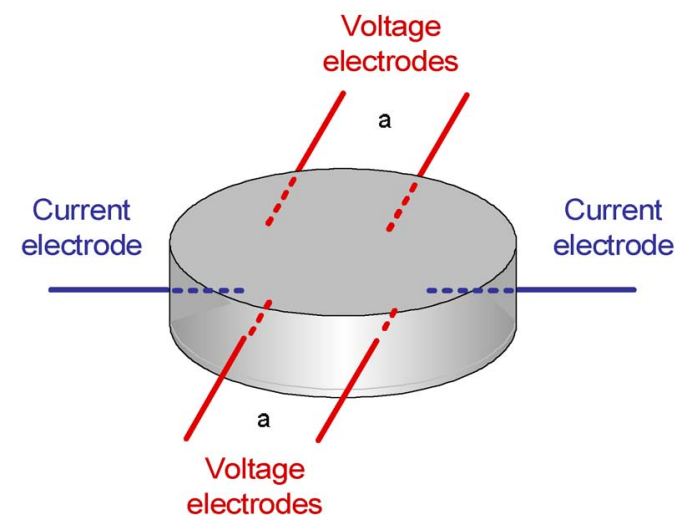

Fig. 4 Specimens used for measurement of electrical resistivity of electrically conductive adhesives, $a=3$ $\mathrm{mm}$

\section{Results ANd Conclusions}

Results are shown in Fig. 5. It has been found that addition of $\mathrm{AgNO}_{3}$ into one component adhesive has had a very low negative influence on an electrical conductivity change; on the other hand, addition of $\mathrm{AgNO}_{3}$ into two components adhesive has improved its conductivity significantly. The reason is another type of initiator used in this adhesive in comparison with the first one.

It has been confirmed for one type of adhesive that addition of $\mathrm{AgNO}_{3}$ into the formulation can improve, under proper conditions, electrical conductivity of adhesive significantly. Therefore it will be paid attention to this direction of research.

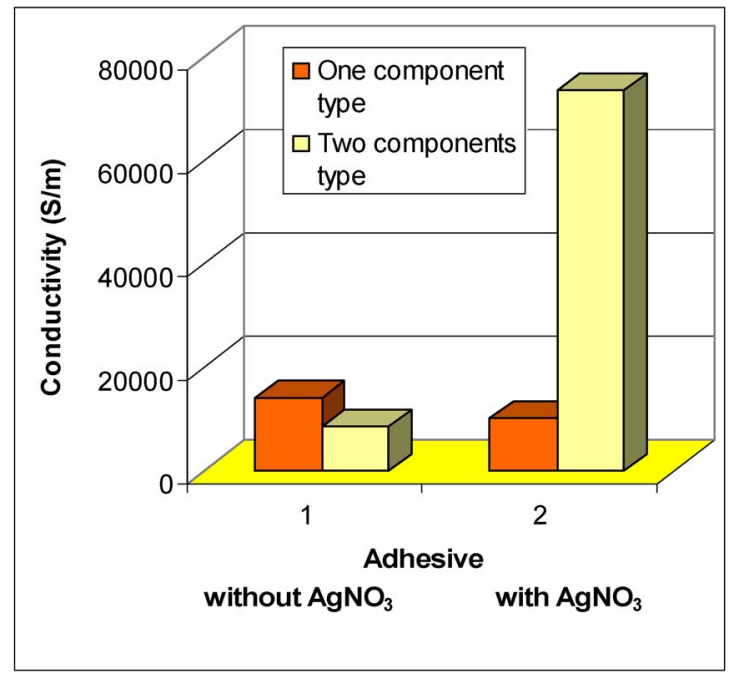

Fig. 5 Electrical conductivity of modified adhesive

\section{REFERENCES}

1. Chen, F. F., Chang, J. P.: Lecture Notes on Principles of Plasma Processing. Kluwer. 2003

\section{ACKNOWLEDGMENT}

The work has been supported by a project

"Diagnostics of Materials", number

MSM6840770021. 